\title{
Werner-Porstmann-Preis zur Förderung der Interventionellen Radiologie
}

In Gedenken an Werner Porstmann (1921 - 1982), einen Pionier der kardiovaskulären Interventionen, schreiben die Deutsche Röntgengesellschaft und die Deutsche Gesellschaft für Interventionelle Radiologie und minimalinvasive Therapie (DeGIR) den Werner-Porstmann-Preis zur Förderung der Interventionellen Radiologie aus.

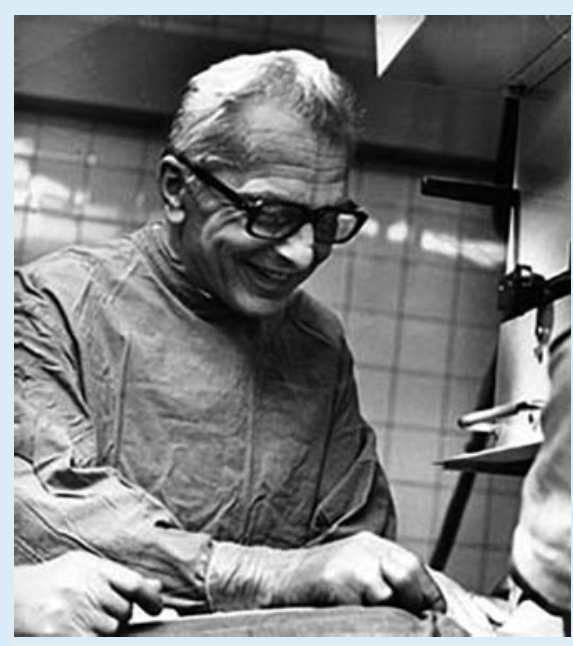

Prof Dr. med. Werner Porstmann (1921 1982). Wegweiser der Forschung in der Diagnostik vaskulärer und kardiovaskulärer Erkrankungen

Er wird jährlich auf dem Deutschen Röntgenkongress im Rahmen der DeGIR-Mitgliederversammlung durch den Vorsitzenden der DeGIR überreicht. Der Preis wird durch die Firma Boston Scientific unterstützt.

\section{Auswahlkriterien}

Der Preis wird jährlich verliehen für die beste wissenschaftliche Arbeit auf dem Gebiet der Interventionellen Radiologie, die in den zwei der Verleihung vorausgehenden Jahren veröffentlicht bzw. zur Veröffentlichung angenommen wurde. Der einreichende Autor muss Mitglied der DRG und der DeGIR sein. Den Hauptteil der zugrundeliegenden Studien sollte der Bewerber hauptsächlich selbstständig durchgeführt haben. Es darf nur eine Publikation eingereicht werden.

\section{Bewerbungsunterlagen und Fristen}

Die Bewerbungsunterlagen enthalten die wissenschaftliche Arbeit, einen kurzen Lebenslauf sowie ein Schriftenverzeichnis der letzten zwei Jahre, an denen der einreichende Autor als Erst- oder Letztautor beteiligt war. Bei Veröffentlichungen mit mehreren Autoren ist der Bewerbung eine Bestätigung des Vorgesetzten beizufügen, die ausweist, dass der einreichende Autor den entscheidenden Anteil an der Veröffentlichung trägt.
Die Bewerbungsunterlagen sind in einfacher schriftlicher Form sowie in elektronischer Form (.doc -Format oder PDF, auf CD gespeichert) bis zum 31. Januar 2018 einzusenden an:

Deutsche Röntgengesellschaft e. V.

Frau Sandra Ermisch

Ernst-Reuter-Platz 10

10587 Berlin

ermisch@drg.de 